\title{
Analyse von Fließgeschwindigkeiten und Abflusstiefen auf verschiedenen Typen von Blockrampen
}

Blockrampen werden oftmals als Ersatz für Schwellen und Abstürze eingesetzt, um gefährlichen Sohlenerosionen entgegenzuwirken und die Fischwanderung zu erleichtern. Um die Durchgängigkeit für Forellen zu gewährleisten, müssen bestimmte hydraulische Kriterien erfüllt sein. Systematische Laborversuche und Feldmessungen auf verschiedenen Typen von Blockrampen haben gezeigt, dass monotone Blockrampen die Kriterien der Forellenmigration nur selten erfüllen. Strukturierte Blockrampen hingegen können bis zu spezifischen Abflüssen von $1 \mathrm{~m}^{3} /(\mathrm{s} \cdot \mathrm{m})$ bzw. $2 \mathrm{~m} /(\mathrm{s} \cdot \mathrm{m})$ auch bei einer Neigung von $10 \%$ bzw. $6 \%$ noch durchgängig sein.

\section{Einleitung}

Gefährliche Sohlenerosionen in Fließgewässern werden oft mit Blockrampen verhindert. Im Vergleich zu Schwellen und Abstürzen erleichtern diese den Fischaufstieg erheblich. Blockrampen müssen aber gewisse hydraulische Kriterien erfüllen, um die Durchgängigkeit für Fische zu gewährleisten. Mit systematischen Laborversuchen wurde für verschiedene Typen von Blockrampen der maximale Abfluss ermittelt, bei dem die hydraulischen Durchgängigkeitskriterien noch eingehalten werden können. Vier verschiedene Ty-

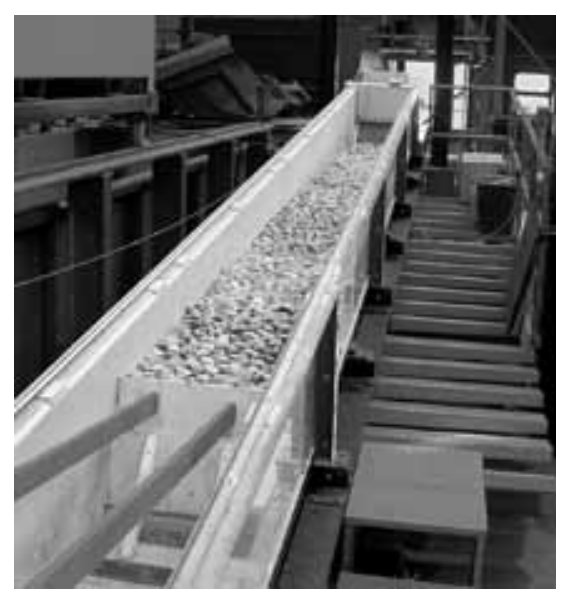

Bild 1: Versuchskanal mit Blockrampe ohne (links) und mit Abfluss (rechts) (Neigung $6 \%$ ) pen von Blockrampen wurden untersucht: eine klassisch gesetzte Rampe mit $10 \%$ Neigung, eine aufgelöste, $6 \%$ geneigte Rampe mit Querriegeln sowie zwei mäanderförmige Rampen mit $10 \%$ und $6 \%$ Neigung. Es wurden statistische und räumliche Verteilungen der Fließgeschwindigkeiten, Abflusstiefen sowie der Verhältnisse von kinetischer Energie zur Abflusstiefe der untersuchten Blockrampen miteinander verglichen. Die erhaltenen Messwerte wurden anschließend mit Feldmessungen auf zwei klassischen Blockrampen ergänzt, die eine betoniert und die andere geschüttet.

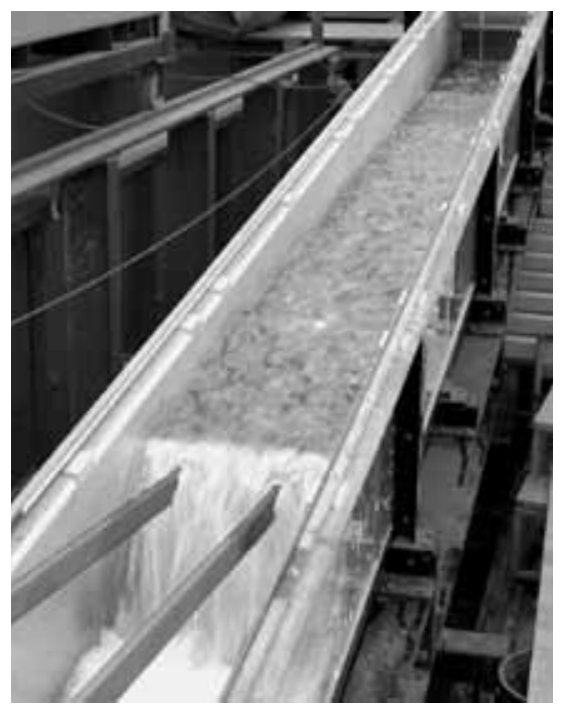

\section{Modellversuche}

\subsection{Versuchseinrichtung und Messverfahren}

Die Versuche wurden in einer 7,7 $\mathrm{m}$ langen, $0,5 \mathrm{~m}$ breiten, $0,5 \mathrm{~m}$ tiefen und um $11 \%$ geneigten, rechteckigen Rinne durchgeführt (Bild 1). Die Morphologie der Rampen wurde mit gebrochenen Steinen ausgelegt (Tabelle 1). Der Abfluss nach dem Einlaufbehälter wurde mit einer 2,5 m langen Einlaufstrecke mit einer Neigung von 3,5\% beruhigt. Danach folgte die eigentliche $3 \mathrm{~m}$ lange Rampe mit einer Neigung von $6 \%$ bzw. $10 \%$.

Die Geschwindigkeiten wurden mit einem kleinen Messflügel gemessen, die Topografie und die Abflusstiefe mit einem Stechpegel. Die Messpunkte waren regelmäßig in einem Raster von 12 × $3 \mathrm{~cm}$ auf der Rampe verteilt. Für strukturreiche Morphologien wurde das Raster auf $6 \times 3 \mathrm{~cm}$ verfeinert. Die Resultate wurden anschließend auf eine Prototyprampe hochgerechnet (Maßstab 1:25), um die Durchgängigkeitskriterien überprüfen zu können.

\subsection{Gemessene}

Durchgängigkeitskriterien

Für die Bestimmung der Durchgängigkeitskriterien der Blockrampen wurde die Forelle ausgewählt. Gemäß DVWK [1] und DWA [3] wurden die maximale Fließgeschwindigkeit $\left(\mathrm{v}_{\max } \leq 2 \mathrm{~m} / \mathrm{s}\right)$, die mini- 
Tab. 1 Granulometrie der verwendeten Blöcke für den Rampenaufbau

(Prototypwerte in Klammern für einen Maßstab 1:25)

\begin{tabular}{|c|c|c|c|c|}
\hline Blockdurchmesser & $\begin{array}{c}D_{m} \\
{[\mathrm{~mm}]} \\
([\mathrm{m}])\end{array}$ & $\begin{array}{c}\mathrm{D}_{30} \\
{[\mathrm{~mm}]} \\
([\mathrm{m}])\end{array}$ & $\begin{array}{c}\mathrm{D}_{65} \\
{[\mathrm{~mm}]} \\
([\mathrm{m}])\end{array}$ & $\begin{array}{c}\mathrm{D}_{90} \\
{[\mathrm{~mm}]} \\
([\mathrm{m}])\end{array}$ \\
\hline Nicht exponierte Blöcke & $36(0,90)$ & $35(0,87)$ & $38(0,94)$ & $40(1,00)$ \\
\hline Exponierte Blöcke & $51(1,26)$ & $49(1,24)$ & $52(1,29)$ & $53(1,33)$ \\
\hline
\end{tabular}

male Abflusstiefe $\left(\mathrm{h}_{\min } \geq 20 \mathrm{~cm}\right)$, minimale Beckentiefen bei Step-pool-step-Systemen $\left(h_{p, \min } \geq 60 \mathrm{~cm}\right)$ sowie maximale Wasserspiegelunterschiede $\left(\Delta_{\mathrm{hmax}} \leq 20 \mathrm{~cm}\right)$ als Kriterien verwendet.

\subsection{Morphologie der untersuchten Blockrampen}

Es wurden vier Rampentypen untersucht, nämlich

- Typ I: Klassisch gesetzte Blockrampe mit monotoner Morphologie (Neigung $10 \%)$.

- Typ II: Aufgelöste strukturierte Blockrampe (Neigung $6 \%$ ); die obere Hälfte der Rampe bestand aus unregelmäßigen Querriegeln mit bis zu 7,7 m Abstand (Bild 2 oben links); die untere Hälfte der Rampe aus regelmäßigen geraden Querriegeln mit 2,8 bis 3,3 $\mathrm{m}$ Abstand gemäß Gebler [2] (Bild 2 oben rechts). Die Gerinnesohle wurde mit Blöcken der feineren Granulometrie ausgelegt, die Querriegel mit der gröberen (Tabelle 1). Die Querriegel haben eine durchschnittliche Höhe von $0,75 \mathrm{~m}$.

- Typ III: Mäanderförmige Blockrampe (Neigung $10 \%$ ). Dieser Typ bestand aus mäanderförmigen Gerinnen, welche durch eine hügelförmige Anordnung der Steinblöcke entstanden. Ihre Erhöhungen und Vertiefungen lagen durchschnittlich $0,4 \mathrm{~m}$ über bzw. unter der mittleren Sohlenlage. Die ersten $25 \mathrm{~m}$ der Rampe bestanden aus zwei Mäandern mit einer gegenseitigen Verschiebung von einer halben Phase. Der Rest der Rampe bestand lediglich aus einem Mäander. Die mittlere Amplitude der Mäander betrug 3,75 $\mathrm{m}$ und die Wellenlänge $17 \mathrm{~m}$.

- Typ IV: Mäanderförmige Blockrampe (Neigung $6 \%$ ); mit Aufbau wie Typ III. Die hügelförmigen Erhöhungen wurden im Vergleich zu Typ III anzahlmäßig vergrößert. Dadurch entstanden über die gesamte Rampenlänge zwei gegenseitig um eine halbe Phase verschobene Mäander (Bild 2 unten). Die Erhöhungen und Vertiefungen lagen durch- schnittlich 0,6 m über bzw. unter der mittleren Sohlenlage. Die Amplitude und Wellenlänge der Mäander war dieselbe wie bei Typ III.

\section{Feldmessungen}

Um Vergleichswerte aus der Natur zu erhalten, wurden auf der Wyna bei Menziken, Schweiz, zwei klassische Blockrampen untersucht (Bild 3). Die Morphologie wurde mit einem Nivelliergerät erfasst. Die Abflusstiefen wurden mit einer Messlatte und die Geschwindigkeiten mit einem elektromagnetischen Messflügel in einem Raster von 1 x $1 \mathrm{~m}$ gemessen. Die erste Rampe entspricht einer klassisch betonierten Blockrampe mit einer mittleren Blockgröße von 0,9 m und einer Neigung von $13 \%$. Die zweite ist eine klassische geschüttete Blockrampe mit einer mittleren Blockgröße von 1,1 m und einer Neigung von $8 \%$. Alle Messwerte wurden entsprechend der Blockgröße mit einem Maßstabsfaktor umgerechnet, um sie mit den Laborrampen vergleichen zu können.

\section{Resultate}

\subsection{Abflusstiefen}

Die Verteilung der Abflusstiefen auf den Rampen widerspiegelt grundsätzlich deren Morphologie, wie dies aus Bild 4a ersichtlich ist. Die Abflusstiefen auf der klassisch gesetzten Blockrampe (Typ I) weisen die schmalste Verteilung aller Rampen auf (Standardabweichung $\pm 0,23 \mathrm{~m}$ ). Die Abflusstiefen der anderen Rampen (Typ II bis Typ IV) haben eine wesentlich breitere, aber untereinander ähnliche Verteilung. Die aufgelöste strukturierte Blockrampe (Typ II) weist eine geringfügig schmalere Streuung $( \pm 0,35 \mathrm{~m})$ auf als die mäanderförmigen Rampen (Typ III und IV) $( \pm 0,41 \mathrm{~m}$ und $\pm 0,40 \mathrm{~m})$. Jedoch haben die Blockrampe mit Querriegeln (Typ II) und die mäanderförmige Blockrampe (Typ IV) mit 0,90 $\mathrm{m}$ und 0,94 $\mathrm{m}$ die größten Mittelwerte. Bei einem spezifischen Abfluss von $\mathrm{q}=1,0 \mathrm{~m}^{3} /(\mathrm{s} \cdot \mathrm{m})$ ist in $89 \%$ aller Becken die minimale Wassertiefe von $0,6 \mathrm{~m}$ gewährleistet. Für den spezifischen Abfluss von $\mathrm{q}=2,7 \mathrm{~m}^{3} /(\mathrm{s} \cdot \mathrm{m})$ sind es gar 97 \%. Die Rampe mit Querriegeln verletzt das Kriterium der minimalen Abflusstiefe von 0,2 $\mathrm{m}$ am stärksten für einen spezifischen Abfluss von $\mathrm{q}=1,0 \mathrm{~m}^{3} /$ $(\mathrm{s} \cdot \mathrm{m})$. Dabei wird die minimale Abflusstiefe noch auf $86 \%$ der Rampenfläche eingehalten. Die übrigen untersuchten Rampentypen haben noch bessere Verhältnisse bezüglich minimaler Abflusstiefe. Die im Feld gemessenen Rampen sind diesbezüglich weniger optimal, da der spezifische
Typ II (Neigung 6\%), unregelmăssige (links) und regelmåssige Querriegel (rechts)

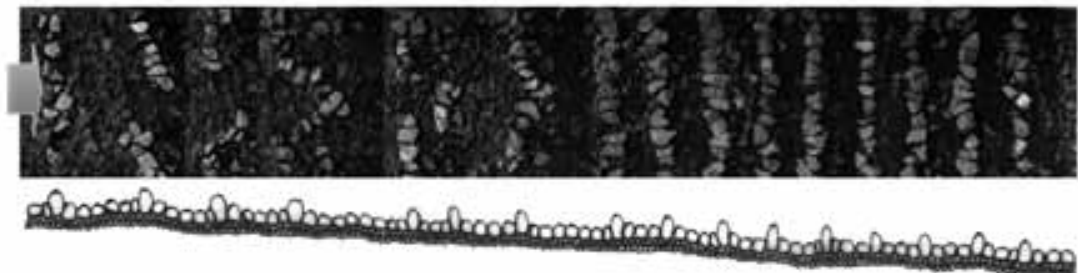

Typ IV (Neigung 6\%)
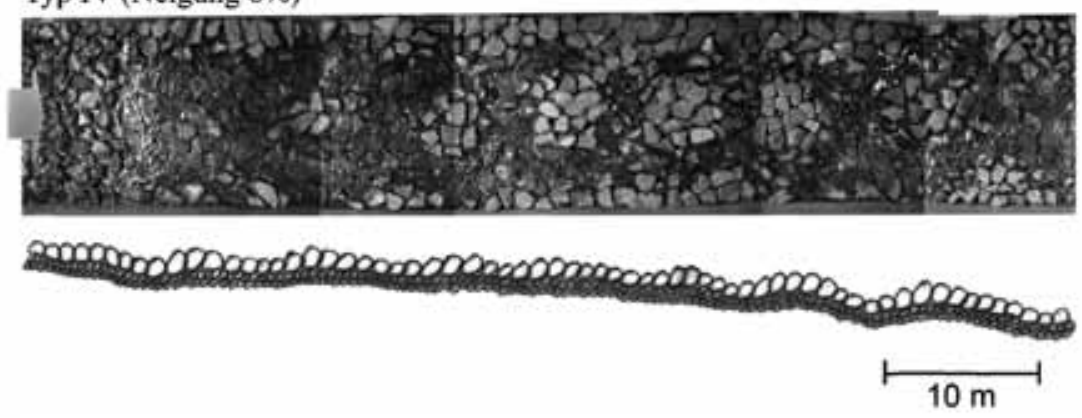

Bild 2: Situation und Längenprofil der Rampen Typ II und Typ IV 

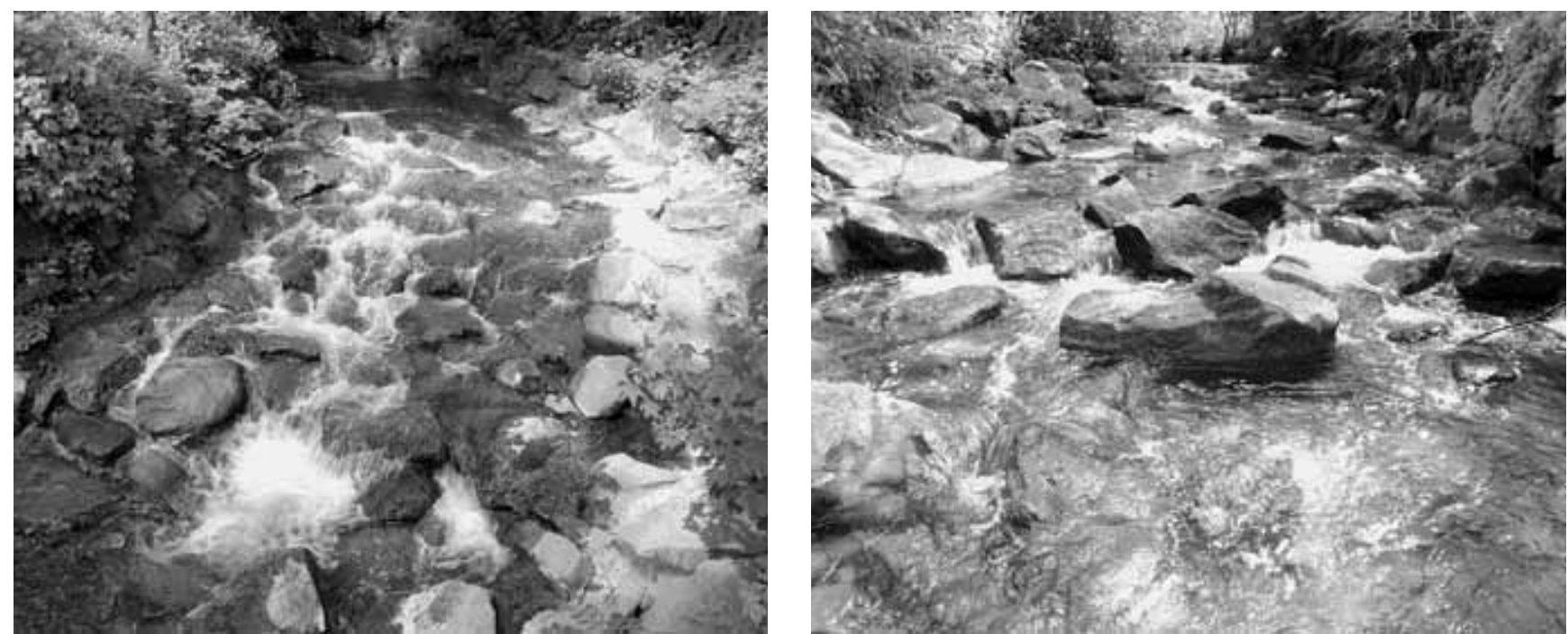

Bild 3: Feldmessungen auf Blockrampen an der Wyna bei Menziken, Schweiz: klassisch betonierte Rampe (links), klassisch geschüttete Rampe (rechts)

Abfluss relativ gering war (Bild 5a). Die mittlere Abflusstiefe der betonierten Rampe lag bei $0,25 \mathrm{~m}$, jene der geschütteten Rampe bei $0,31 \mathrm{~m}$ mit Streuungen von $\pm 0,25 \mathrm{~m}$ respektive $\pm 0,22 \mathrm{~m}$. Auf $41 \%$ der Oberfläche der betonierten Rampe ist das Kriterium der minimalen Abflusstiefe von 0,2 m erfüllt. Bei der geschütteten Rampe ist das auf $60 \%$ der Oberfläche der Fall. Die geringen Abflusstiefen sind somit für die Durchgängigkeit der betonierten Rampe maßgebend (Bild 7 links).

\subsection{Fließgeschwindigkeiten}

Wie erwartet erzeugt die klassisch gesetzte Rampe (Typ I) mit 4,2 m/s die größte mittlere Fließgeschwindigkeit (Bild 4b) mit der geringsten Streuung aller Rampen $( \pm 0,61 \mathrm{~m} / \mathrm{s})$. Die breiteste Streuung $( \pm 1,31 \mathrm{~m} / \mathrm{s})$ wird auf der mäanderförmigen Rampe (Typ III) beobachtet. Ihre mittlere Fließgeschwindigkeit ist mit 3,2 m/s etwas höher als jene der Rampe mit Querriegeln (Typ II) und der mäanderförmigen Rampe (Typ IV), welche beide bei $3,0 \mathrm{~m} / \mathrm{s}$ liegen. In Bild 6 wurden die Flächen mit dem erfüllten Durchgängigkeitskriterium $(\mathrm{v} \leq 2 \mathrm{~m} / \mathrm{s})$ durch eine dicke Linie umrandet. Je größer die umrandeten Flächen, desto eher ist die Rampe durchgängig. Die Rampe mit regelmäßigen Querriegeln (Bild 6 oben rechts) ist eher durchgängig als jene mit unregelmäßigen Querriegeln (Bild 6 oben links). Bei Typ IV ist ein Korridor in der Rampenmitte sowie entlang den Ufern günstig für die Forellendurchgängigkeit (Bild 6 unten). Die im Feld gemessenen Rampen erfüllen das Kriterium der maximalen Fließgeschwindigkeit von $2 \mathrm{~m} / \mathrm{s}$ am Besten, da der spezifische Abfluss relativ gering war (Bild 7). Auf $94 \%$ der Oberfläche der betonierten Rampe lie-
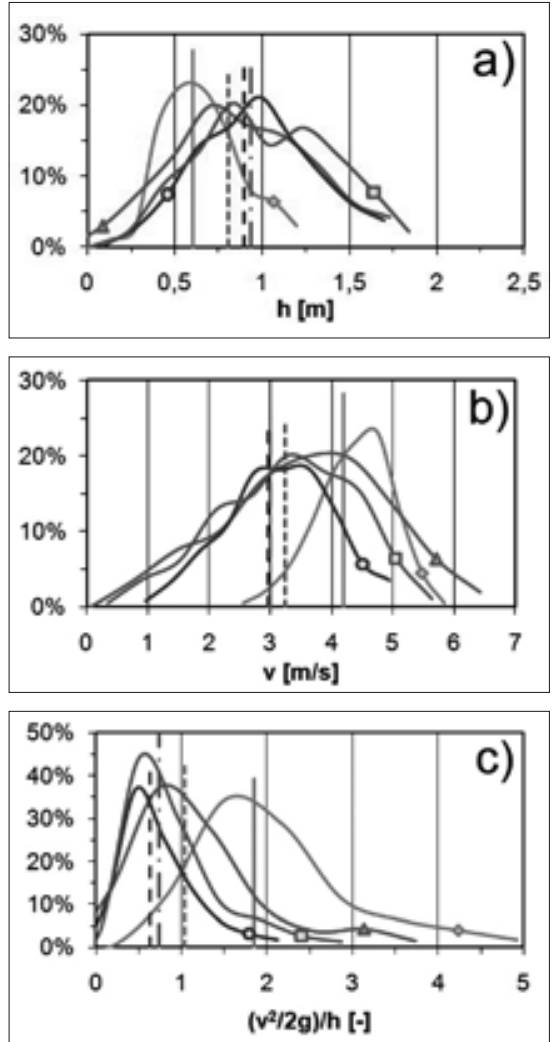

$\multimap$ Typ I (Neigung 10\%) - Mittelwert - -Typ III (Neigung 10\%) --- Mittelwert

Bild 4: Im Labor gemessene Abflussverhältnisse auf einer klassisch gesetzten (Typ I), aufgelöst strukturierten, mit Querriegeln versehenen (Typ II) und auf zwei mäanderförmigen Blockrampen (Typ III, Typ IV): a) Abflusstiefen, b) Fließgeschwindigkeiten, c) Verhältnisse von kinetischer Energie zur Abflusstiefe; jeweils spezifischer Abfluss von $2,7 \mathrm{~m}^{3} /(\mathrm{s} \cdot \mathrm{m})$, Auftretenswahrscheinlichkeit (links) und Überschreitungswahrscheinlichkeit (rechts). 

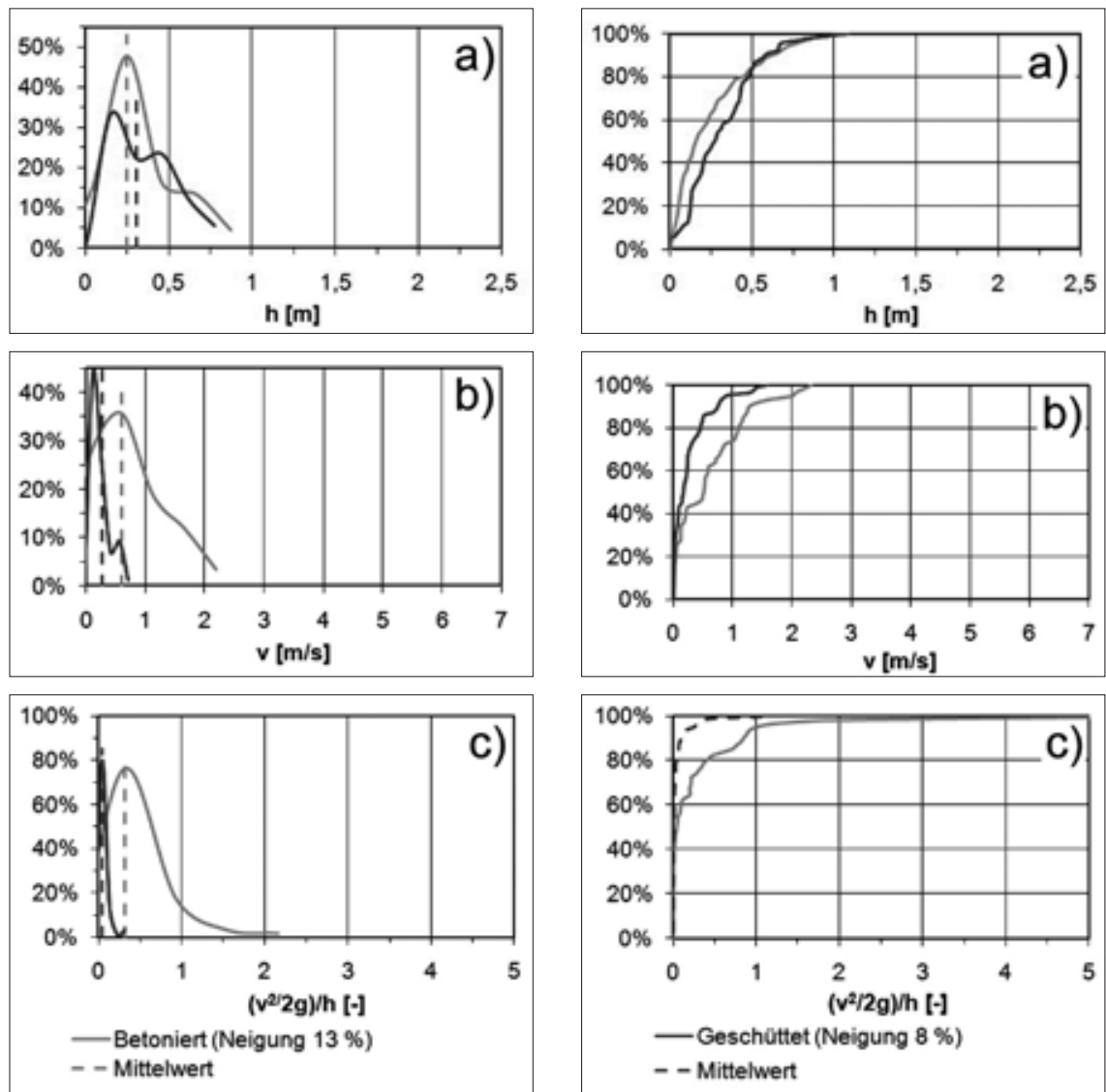

tonierten Rampe beträgt das mittlere Verhältnis von kinetischer Energie zur Abflusstiefe 0,31 und auf der geschütteten Rampe 0,04.

\section{Schlussfolgerungen}

Die klassisch gesetzte Blockrampe ist sowohl für eine Neigung von $6 \%$ als auch für $10 \%$ selbst bei kleinen spezifischen Abflüssen nicht durchgängig für Forellen. Die Rampe mit regelmäßigen Querriegeln gemäß Gebler [2] ist bei einer Neigung von $6 \%$ bis zu einem spezifischen Abfluss von $2 \mathrm{~m}^{3} /(\mathrm{s} \cdot \mathrm{m})$ durchgängig. Falls die Riegel unregelmäßig angeordnet sind, kann die Durchgängigkeit bis zu einem spezifischen Abfluss von $1 \mathrm{~m}^{3} /(\mathrm{s} \cdot \mathrm{m})$ gewährleistet werden.

Als Alternative zur klassischen gesetzten Blockrampe wurde die mäanderförmige Morphologie entwickelt. Die hügelartige Oberfläche erzeugt eine breite Geschwindigkeitsverteilung, was den Fischaufstieg erleichtert. Deshalb ist der Forellenaufstieg bei einer Neigung von $10 \%$ bis zu einem spezifischen Abfluss von $1 \mathrm{~m}^{3} /$ $(\mathrm{s} \cdot \mathrm{m})$ und bei der Neigung von $6 \%$ bis $\mathrm{zu}$ einem spezifischen Abfluss von 1,5 $\mathrm{m}^{3}$ / $(\mathrm{s} \cdot \mathrm{m})$ gewährleistet.

Die mit Feldmessungen untersuchte betonierte Rampe mit der Neigung von $13 \%$ und einem kleinen spezifischen Abfluss von $0,07 \mathrm{~m}^{3} /(\mathrm{s} \cdot \mathrm{m})$ erfüllte wegen den $\mathrm{zu}$ geringen Abflusstiefen die Kriterien der Durchgängigkeit nicht. Hingegen war die im Feld untersuchte geschüttete Rampe mit $8 \%$ Neigung für den gemessenen spezifischen Abfluss von $0,1 \mathrm{~m}^{3} /(\mathrm{s} \cdot \mathrm{m})$ bereits durchgängig.

Zusammenfassend kann gesagt werden, dass gleichmäßige monotone Blockrampen die Durchgängigkeitskriterien für Fo-

\subsection{Verhältnisse von kinetischer Energie zur Abflusstiefe}

Das Verhältnis der kinetischen Energie zur Abflusstiefe $\left(v^{2} / 2\right.$ g)/h kann auch einen Anhaltspunkt für die Fischdurchgängigkeit geben. Es nimmt für höhere spezifische Abflüsse zu (Bild 4c, Bild 5c) und weist auf der klassisch gesetzten Rampe (Typ I) mit 1,9 den höchsten Mittelwert und die breiteste Streuung $( \pm 1,26)$ aller Rampen auf. Bezüglich Fischdurchgängigkeit gilt, je geringer das Verhältnis der kinetischen Energie zur Abflusstiefe, desto besser die Fischdurchgängigkeit. $95 \%$ aller Messpunkte, welche gleichzeitig das Kriterium der maximalen Fließgeschwindigkeit $(\leq 2 \mathrm{~m} / \mathrm{s})$ als auch der minimalen Abflusstiefe ( $\geq 0,2 \mathrm{~m}$ ) erfüllen,

ergeben Verhältnisse von kinetischer Energie zur Abflusstiefe zwischen 0 und 0,45. Man kann deshalb davon ausgehen, dass Verhältnisse größer als 0,45 für die Durchgängigkeit problematisch sind. Die entsprechenden Mittelwerte aus den Feldmessungen liegen um einiges tiefer als jene der Modellversuche. Auf der be-
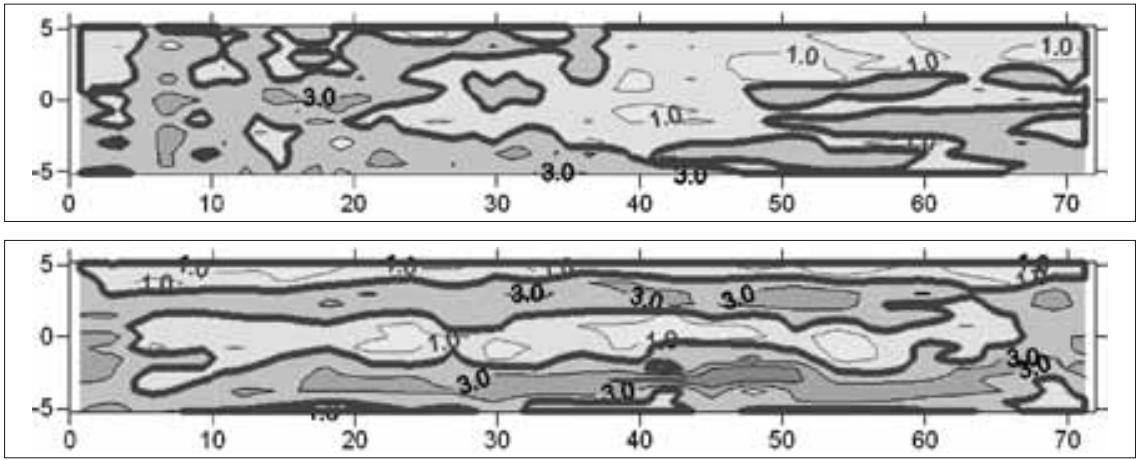

Bild 6: Fließgeschwindigkeiten in $\mathrm{m} / \mathrm{s}$ für den spezifischen Abfluss von 1,5 $\mathrm{m}^{3} /(\mathrm{s} \cdot \mathrm{m})$ von Typ II (oben) und Typ IV (unten), dabei umrandet die dicke Linie Bereiche mit Fließgeschwindigkeiten $\leq 2 \mathrm{~m} / \mathrm{s}$ 

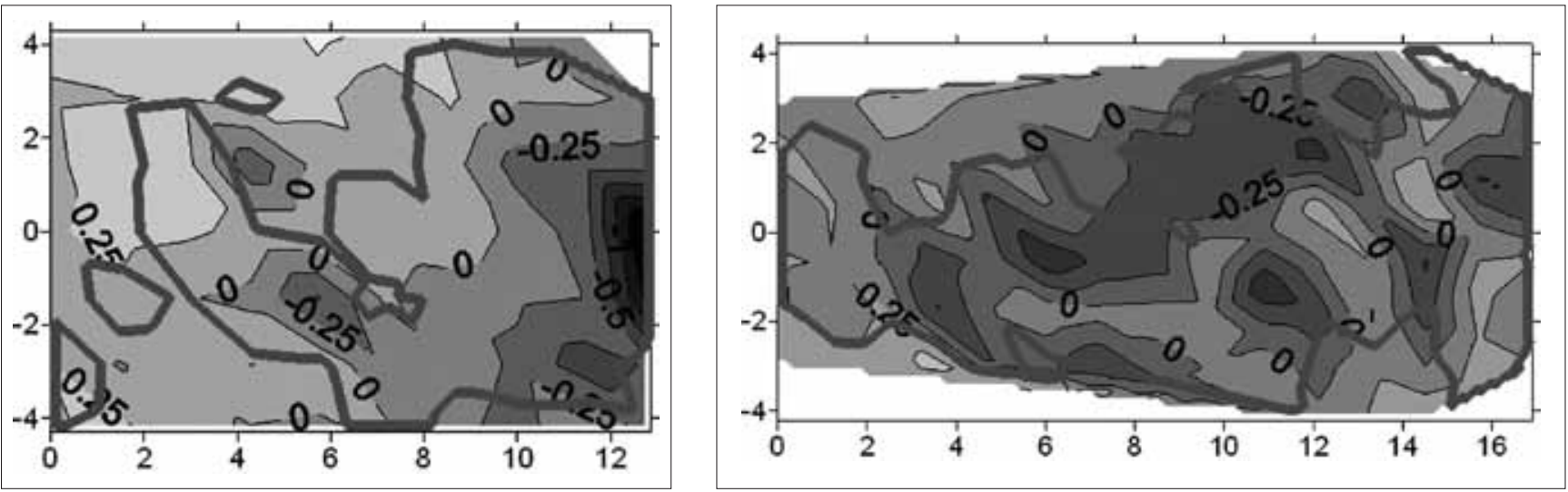

Bild 7: Morphologie der klassischen betonierten Blockrampe (links) und der klassischen geschütteten Blockrampe (rechts) aus den Feldmessungen an der Wyna mit den entsprechenden spezifischen Abflüssen von $0,07 \mathrm{~m}^{3} /(\mathrm{s} \cdot \mathrm{m})$ und $0,1 \mathrm{~m} /(\mathrm{s} \cdot \mathrm{m})$, dabei umrandet die dicke Linie Korridore, bei denen die Kriterien für die Fischdurchgängigkeit $(v \leq 2 \mathrm{~m} / \mathrm{s}, \mathrm{h} \geq 0,2 \mathrm{~m})$ erfüllt sind

rellen kaum erfüllen. Strukturierte Blockrampen sind wesentlich besser geeignet, indem optimale Verhältnisse für den Forellenaufstieg bei Neigungen bis $6 \%$ für spezifische Abflüsse bis maximal $2 \mathrm{~m}^{3} /$ $(\mathrm{s} \cdot \mathrm{m})$ und bei Neigungen bis $10 \%$ für spe-

zifische Abflüsse bis maximal $1 \mathrm{~m}^{3} /(\mathrm{s} \cdot \mathrm{m})$ gewährleistet werden können.

\section{Hinweis}

Die vorliegende Untersuchung wird im Rahmen des Forschungsprojektes Integ-

Markus Studer and Anton Schleiss

\section{Velocity and Water Depth Analysis on Different Types of Block Ramps}

Block ramps are often used as an alternative for sills to protect rivers against dangerous bed erosion and to enhance fish migration. To ensure fish passage, hydraulic criteria must be fulfilled. A systematic laboratory study was performed in order to measure these hydraulic criteria fixed for trouts. Statistical and spatial distributions of velocities, water depths as well as the ratios of water depth and velocity head were compared for different types of block ramps and completed with field measurements on two classical ramp structures. The results have revealed that uniform block ramps can rarely satisfy the criteria for fish migration. Structured block ramps are more efficient. Nevertheless optimum conditions for fish migration on a ramp slope of $6 \%$ and $10 \%$ can be reached only for specific discharges below $2 \mathrm{~m}^{3} /(\mathrm{s} \cdot \mathrm{m})$ and $1 \mathrm{~m}^{3} /(\mathrm{s} \cdot \mathrm{m})$ respectively.

Маркус Штудер и Антон Шляйсс

\section{Анализ скорости потока и стоковых глубин на блоковых рампах различного типа}

Блоковые рампы часто используются как альтернатива порогам и перепадам с целью противодействия опасной эрозии основания и облегчения процесса миграции рыбы. Для обеспечения прохода форели должны быть выполнены определенные, соответствующие законам гидравлики критерии. Систематические лабораторные испытания и геодезические измерения на блоковых рампах различного типа показали, что блоковые рампы монотипа практически не соответствуют условиям миграции форели. Блоковые рампы структурного типа, напротив, проходимы даже на специфических стоках при 1 м3/(с - м) или 2 м3/(с · м), в том числе и при уклоне от $10 \%$ до $6 \%$.

rales Flussgebietsmanagement vom Bundesamt für Umwelt (BAFU), Schweiz, unterstützt. Die Feldmessungen wurden in Zusammenarbeit mit Denise Weibel, Eewag, durchgeführt.

\section{Autoren}

Dipl.-Ing. Markus Studer

Prof. Dr. Dipl.-Ing. Anton Schleiss

Laboratoire de Constructions Hydrauliques (LCH)

Ecole Polytechnique Fédérale de Lausanne (EPFL)

Station 18

1015 Lausanne, Schweiz

anton.schleiss@epfl.ch

\section{Literatur}

[1] DVWK (Hrsg.): Fischaufstiegsanlagen - Bemessung, Gestaltung, Funktionskontrolle. Merkblätter zur Wasserwirtschaft. In: DVWKMerkblätter (1996), Nr. 232.

[2] Gebler, R. J.: Sohlrampen und Fischaufstiege. Walzbachtal: Eigenverlag, 1991.

[3] DWA (Hrsg.): Fischaufstiegsanlagen und fischpassierbare Bauwerke - Gestaltung, Bemessung, Qualitätssicherung. In: DWA Merkblatt (2010) Nr. M 509 (Entwurf).

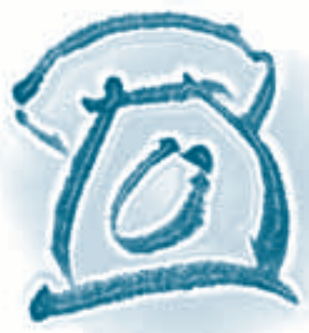

\section{Anzeigen-Service (0611) 7878338}

